\title{
VIGILANCIA DE LA SENSIBILIDAD A PENICILINA EN STREPTOCOCCUS AGALACTIAE RECUPERADOS DE EMBARAZADAS ENTRE LAS 35-37 SEMANAS DE GESTACIÓN.
}

\author{
SURVEILLANCE OF SENSITIVITY TO PENICILLIN IN STREPTOCOCCUS AGALACTIAE RECOVERED \\ FROM PREGNANT WOMEN BETWEEN 35-37 WEEKS OF GESTATION.
}

\author{
Lucía F. Müller Velázquez'; Fernando J. Bobadilla ${ }^{2,3}$; Marina G. Novosak ${ }^{2,3}$; lliana J. Cortese ${ }^{2,3}$; Margarita \\ E. Laczeski $i^{2,3^{*}}$
}

\section{Resumen}

Streptococcus agalactiae (SGB) es causa de infecciones severas en menores de tres meses. Meningitis, neumonía y sepsis son los principales cuadros en estos niños. Estas infecciones se encuentran entre las más graves que puede sufrir un individuo en sus primeras doce horas de vida. El niño adquiere la infección por transmisión vertical de la madre colonizada. Para prevenir la enfermedad neonatal se recomienda penicilina como droga de elección en la profilaxis intraparto (PIP) en embarazadas colonizadas. Sin embargo, actualmente se han detectado cepas con sensibilidad disminuida a penicilina por lo que resulta importante realizar la vigilancia de la sensibilidad al mismo para asegurar su utilidad durante la profilaxis. El objetivo de este trabajo fue determinar la sensibilidad a penicilina en cepas de SGB recuperados de mujeres embarazadas de 35-37 semanas de gestación. Se estudiaron 96 aislamientos y se determinó la sensibilidad por método epsilométrico Etest $®$ (LIOFILCHEM, Italia), siguiendo las recomendaciones del Clinical Laboratory Standards Institute (CLSI). Se obtuvo la Concentración Inhibitoria Mínima (CIM) para cada aislamiento bacteriano. El 100\% (96) de las cepas estudiadas fue sensible a penicilina con valores de CIM comprendidos entre 0,012 y 0,094 $\mu \mathrm{g} \mathrm{mL-1.} \mathrm{Estos}$ resultados indican que penicilina sigue siendo el antimicrobiano de elección durante la profilaxis intraparto, para la prevención de la enfermedad neonatal causada por SGB en nuestra región. Se destaca la importancia de la vigilancia epidemiológica de la sensibilidad a penicilina y a otros antimicrobianos para alertar sobre nuevos mecanismos de resistencia y adecuar estrategias de tratamiento.

Palabras clave: streptococcus agalactiae; SGB; antimicrobiano; penicilina; profilaxis intraparto.

\section{Abstract}

Streptococcus agalactiae (GBS) cause severe infections in newborns under three months. Meningitis, pneumonia and sepsis are the main infectious diseases in these children. These infections are among the most serious that an individual can suffer in his first twelve hours of life. The child acquires the infection by vertical transmission of the colonized mother. To prevent neonatal disease, penicillin is recommended as the drug of choice for intrapartum prophylaxis (PIP) in pregnant women colonized. However, strains with decreased susceptibility to penicillin have been detected so it is important to monitor the susceptibility to penicillin to ensure its usefulness during prophylaxis. The aim of this study was to determine the sensitivity to penicillin in GBS strains recovered from pregnant women with 35-37 weeks of gestation. Ninety-six isolates were studied and sensitivity was determined by the epsilometric method Etest ${ }^{\circ}$ (LIOFILCHEM, Italy), following the recommendations of the Clinical Laboratory Standards Institute (CLSI). Minimum Inhibitory Concentration (MIC) was obtained for each bacterial isolation. 100\% (96) of the strains studied were sensitive to penicillin with MIC values between 0.012 and $0.094 \mu \mathrm{gL}-1$. These results indicate that penicillin remains the antimicrobial of choice during intrapartum prophylaxis, for the prevention of neonatal disease caused by GBS in our region. The importance of epidemiological surveillance of sensitivity to penicillin and other antimicrobials is highlighted in order to alert new resistance mechanisms and to adapt treatment strategies.

Key words: streptococcus agalactiae; GBS; antimicrobial; penicillin; intrapartum prophylaxis

\footnotetext{
1 Cátedra de Microbiología General, Departamento de Microbiología, Facultad de Ciencias y Tecnología, Universidad Nacional de Itapúa. Encarnación, Paraguay.

2 Cátedra de Bacteriología, Departamento de Microbiología, Facultad de Ciencias Exactas, Químicas y Naturales. Universidad Nacional de Misiones. Posadas, Argentina.

3 Laboratorio de Biotecnología Molecular, Instituto de Biotecnología Misiones "Dra. María Ebe Reca", Facultad de Ciencias Exactas, Químicas y Naturales. Universidad Nacional de Misiones. Posadas, Argentina.

4 Email de contacto: mlaczeski@gmail.com ó melaczeski@fceqyn.unam.edu.ar
} 


\section{Introducción}

Streptococcus agalactiae grupo B (SGB) es un coco Gram positivo que forma parte de la microbiota normal del tracto gastrointestinal y genitourinario, sitios que constituyen reservorios en el humano y desde donde coloniza la vagina y con frecuencia el tracto urinario de cerca del $20 \%$ de mujeres embarazadas y no embarazadas ${ }^{(1)}$.

Streptococcus agalactiae causa meningitis, neumonía y sepsis en neonatos con alta morbilidad y mortalidad, siendo responsable de las infecciones más graves que puede presentar un individuo en sus primeras horas de vida ${ }^{(2)}$. Se asocia también a infecciones urinarias, infecciones de heridas quirúrgicas post-cesárea, sepsis puerperal y actualmente en pacientes con enfermedades crónicas subyacentes, relacionado a infecciones invasivas severas, como neumonía, peritonitis, endocarditis, meningitis, osteomielitis, artritis, celulitis necrotizante y fascitis necrotizante $e^{(2,3,4)}$

La infección perinatal causada por SGB puede ser de inicio precoz, dentro de los primeros 6 días de vida comenzando generalmente dentro de las 12 horas del nacimiento o, de inicio tardío, luego de la primera semana de vida y hasta los tres meses. La primera se caracteriza por tener una presentación tórpida de distrés respiratorio, apneas, neumonía, meningitis, sepsis y shock. La segunda se presenta con elevada incidencia de meningitis y bacteriemia ${ }^{(5)}$.

La colonización con SGB en los recién nacidos se produce durante el parto, a partir del tracto genital materno colonizado o en el útero, por vía ascendente, siendo la tasa de transmisión vertical del $50 \%$ y de ellos, $1-2 \%$ desarrollan sepsis neonatal ${ }^{(6,7)}$.

En este sentido, el Centro de Control y Prevención de Enfermedades de Atlanta, EE.UU. $(C D C)^{(8)}$ sugiere como medida de prevención de la enfermedad neonatal, la búsqueda de la portación de SGB durante las 35-37 semanas de embarazo y la instauración de la Profilaxis IntraParto (PIP) con antimicrobianos a las mujeres colonizadas. En la República Argentina ${ }^{(9)}$ en el año 2008 se sanciona la Ley Nacional $N^{\circ} 26.369$ que adhiere a las recomendaciones del CDC y establece la obligatoriedad de la búsqueda de portación materna de SGB en toda gestante entre las semanas 35 y 37 de embarazo.

Además de la búsqueda microbiológica de SGB en la mujer embarazada, ciertos factores de riesgo se encuentran asociados a la infección perinatal, los cuales una vez detectados orientan a la prevención a través de la PIP, ellos son: antecedente previo de recién nacido con sepsis precoz por SGB, corioamnionitis, bacteriuria por
SGB en el embarazo actual, parto pretérmino (menor a 37 semanas), fiebre materna durante el trabajo de parto $\left(\geq 38^{\circ} \mathrm{C}\right)$, parto prolongado con rotura prematura de membrana ${ }^{(10)}$.

Detectada la colonización materna y/o los factores de riesgo, las políticas de manejo, indican la administración de profilaxis antimicrobiana intraparto (PIP) para prevenir la enfermedad perinatal por SGB.

Streptococcus agalactiae ha sido considerado universalmente sensible a penicilina, por lo que la penicilina $G$ y otros antibióticos beta-lactámicos, como ampicilina, constituyen los antimicrobianos de elección para instaurar la PIP y el tratamiento de las infecciones causadas por SGB ${ }^{(11-14)}$. De hecho, ningún criterio de "resistencia a penicilina" ha sido establecido hasta la fecha por el CLSI (Clinical Laboratory Standard Institute).

Las primeras guías de consenso para la prevención de la enfermedad neonatal fueron publicadas en el año 1996 por el CDC y modificadas en el año $2002^{(15)}$ en el que se recomienda la administración de PIP a todas aquellas embarazadas que presentaran un cultivo positivo para SGB realizado entre las 35 y 37 semanas de gestación aún sin que presenten los demás factores de riesgo citados. La administración de penicilina debe hacerse por vía endovenosa y deben ser recibidos por la madre al menos 4 horas antes del parto, para alcanzar concentraciones adecuadas $^{(15,16)}$.

intra-amnióticas

A pesar de la marcada sensibilidad de SGB a penicilina y ampicilina, varios estudios indican respuestas clínicas pobres a la terapia estándar y recaída en infecciones por SGB en neonatos y adultos. Así, algunos investigadores como Betriu y col $(1994)^{(17)}$, Kimura y col. $(2008)^{(13)}$ y Dahesh y col $(2008)^{(14)}$, demostraron fenotípicamente la tolerancia a penicilina en estos microorganismos y sugirieron que esto contribuye al fracaso de los tratamientos. Además, también se han caracterizado a cepas de SGB con sensibilidad reducida a penicilina desde el punto de vista molecular, adjudicándoles dicha propiedad a la sustitución de aminoácidos adyacentes a los dominios conservados SSN y KSG de la Proteína de Unión a Penicilina 2X (PBP 2X) $)^{(14,18)}$.

Para comprender la participación de estas sustituciones aminoacídicas, se debe recordar que la biosíntesis del peptidoglucano se da en sucesivos pasos. La etapa final de este proceso es la transpeptidación de la molécula de peptidoglucano en desarrollo con la formación de enlaces cruzados entre las cadenas lineales para formar una malla bi o tridimensional. Estos enlaces se establecen entre los aminoácidos del pentapéptido, concretamente entre el aminoácido 
en posición 3 (que siempre es dibásico y tiene un grupo amino libre) y el residuo D-ala en posición 4. Esto hace que se desplace el resto de D-ala en posición 5. Las enzimas que catalizan esta etapa se llaman transpeptidasas ${ }^{(19)}$.

Una segunda enzima, la D-ala-carboxipeptidasa, elimina los restos $D$-ala terminales de cualquier pentapéptido que no está comprometido en el entrecruzamiento. Estas dos enzimas, transpeptidasas y carboxipeptidasas, son inhibidas por los antibióticos beta-lactámicos y forman parte de una familia conocida como Proteínas de Unión a Penicilina (Penicillin Binding Protein- PBP). Las PBPs se numeran según su peso molecular y la proteína 1 tiene el peso molecular más alto ${ }^{(20)}$.

La actividad de los beta-lactámicos se debe principalmente a la inhibición que producen a partir de la reacción de transpeptidación. La estructura de estos antibióticos, en su anillo betalactámico, es similar a la del dipéptido D-ala-Dala que es el sustrato natural reconocido por las transpeptidasas en la reacción de entrecruzamiento de la mureína. Al contrario de lo que ocurre con el sustrato natural, los betalactámicos se unen a la transpeptidasa formando un enlace covalente con una serina de su centro activo, lo que produce la inactivación irreversible de la enzima ${ }^{(19)}$.

Un mecanismo importante de resistencia de las bacterias a los beta-lactámicos es la alteración de estas PBPs ${ }^{(20)}$.

Las alteraciones existentes en las regiones cercanas a los sitios activos de la PBP2X (sitios activos: aminoácidos 402-404 y 552-554) son los determinantes críticos responsables de la susceptibilidad reducida a los beta-lactámicos ${ }^{(13)}$. Las sustituciones aminoacídicas encontradas en la PBP2X de los PRGBS pueden ser clasificadas en dos tipos: 1) Q557E (sustitución del aminoácido Glutamina por ácido Glutámico en la posición 557) y 2) V405A (sustitución de Valina por Alanina en la posición 405$)^{(13,14,18)}$.

Los estudios mencionados sobre la sensibilidad disminuida a penicilina detectada por investigadores como Kimura y col. $(2008)^{(13)}$, Dahesh y col. $(2008)^{(14)}$, Betriu y col. $(2015)^{(17)}$ nos llevan a plantear la carencia de datos sobre la vigilancia de la sensibilidad a penicilina en la provincia de Misiones, siendo este antibiótico, el de elección como primera línea en la PIP y en el tratamiento de otras infecciones por SGB, reforzando la necesidad de conocerlos para contribuir a la prevención de la severa enfermedad neonatal producida por SGB dada la relevancia que tiene como desafío a los paradigmas terapéuticos establecidos en la actualidad. Por lo tanto, el objetivo de este trabajo fue determinar la sensibilidad a penicilina en cepas de SGB recuperados de mujeres entre las 35-37 semanas de gestación y concurrentes al Hospital Público "Dr. Ramón Madariaga" de la ciudad de Posadas.

\section{Materiales y métodos}

\section{Cepas bacterianas estudiadas}

Se estudiaron 96 cepas de SGB recuperados de hisopos vagino-rectales de mujeres embarazadas con 35-37 semanas de gestación, no hospitalizadas y concurrentes al servicio de Ginecología y Obstetricia del Hospital "Dr. Ramón Madariaga" de la ciudad de Posadas entre enero de 2010 y julio de 2014.

Las cepas fueron identificadas con pruebas bioquímicas convencionales descriptas en la bibliografía ${ }^{(21)}$ : coloración de Gram, hemólisis en agar sangre, prueba de catalasa, prueba de bilisesculina, hidrólisis de hipurato, prueba de CAMP y serológicas de grupo (Phadebact Streptococcus Test-Boule Diagnostics ABE-Suecia).

Se utilizaron controles negativos y positivos para cada prueba. Como control positivo de género y especie, se utilizó la cepa de Streptococcus agalactiae (SGB) ATCC 6394 y para la prueba de catalasa, Staphylococcus aureus subesp. aureus Rosembach ATCC 25923. Como control negativo fueron utilizadas las cepas de Enterococcus faecalis ATCC 29212 (para pruebas de CAMP y Serología) y de Escherichia coli ATCC 25922 (para prueba de hidrólisis de hipurato).

Una vez identificadas las cepas de SGB fueron conservadas en leche descremada al $20 \%$ a $80^{\circ} \mathrm{C}$ hasta la realización de las pruebas de susceptibilidad a penicilina.

\section{Pruebas de sensibilidad por el método de la concentración inhibitoria mínima (CIM)}

Se determinó la susceptibilidad a penicilina (PEN) según el método de la Concentración Inhibitoria Mínima (CIM) en placa de agar Mueller-Hinton suplementado con sangre ovina al $5 \%$, siguiendo las recomendaciones del Clinical Laboratory Standards Institute (CLSI) ${ }^{(22)}$.

Se utilizaron tiras con un gradiente de concentración predefinido de Penicilina $G$ de 0,002 a $32 \mu \mathrm{g} \mathrm{mL} \mathrm{m}^{-1}$, con 15 diluciones de lectura (método epsilométrico Etest $®$-LIOFILCHEM, Italia).

Para la interpretación de los resultados se tomaron los puntos de corte establecidos por el $\mathrm{CLSI}^{(23)}$ que determina sensibilidad a penicilina con $\mathrm{CIM} \leq 0,12 \mu \mathrm{g} \mathrm{mL}-1$. Como control de 
calidad del procedimiento se utilizó a Streptococcus pneumoniae ATCC 49619.

\section{Análisis estadístico}

Los valores de CIM obtenidos fueron analizados estadísticamente utilizando la aplicación Statgraphics Centurion XVII a fin de obtener una mejor descripción de los datos.

\section{Resultados:}

El $100 \%$ (96) de las cepas estudiadas fueron sensibles a penicilina según el criterio de CIM establecido por CLSI con valores comprendidos entre 0,012 y $0,094 \mu \mathrm{gL}^{-1}$ (Tabla 1 y Figura 1).

Tabla 1: Resumen estadístico de resultados de CIM para las 96 cepas de Streptococcus agalactiae.

\begin{tabular}{ll}
\hline Recuento & 96 \\
\hline Promedio & 0,0409792 \\
\hline Desviación Estándar & 0,0141912 \\
\hline Coeficiente de Variación & $34,6302 \%$ \\
\hline Mínimo & 0,012 \\
\hline Máximo & 0,094 \\
\hline Rango & 0,082 \\
\hline
\end{tabular}

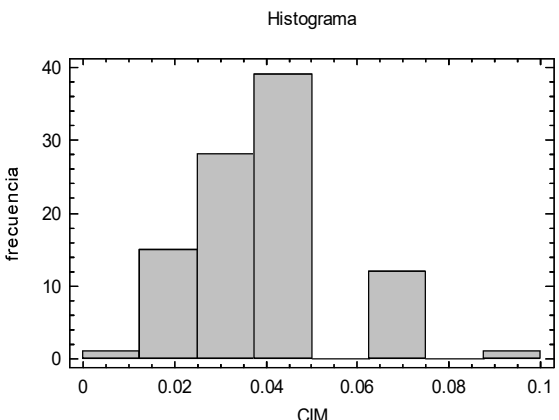

Figura 1. Distribución de frecuencias de los valores de CIM de 96 cepas de Streptococcus agalactiae

En la Figura 2 se observa la CIM por el método epsilométrico de un aislamiento de SGB sensible a penicilina $(0,047 \mu \mathrm{g} \mathrm{mL}-1)$.

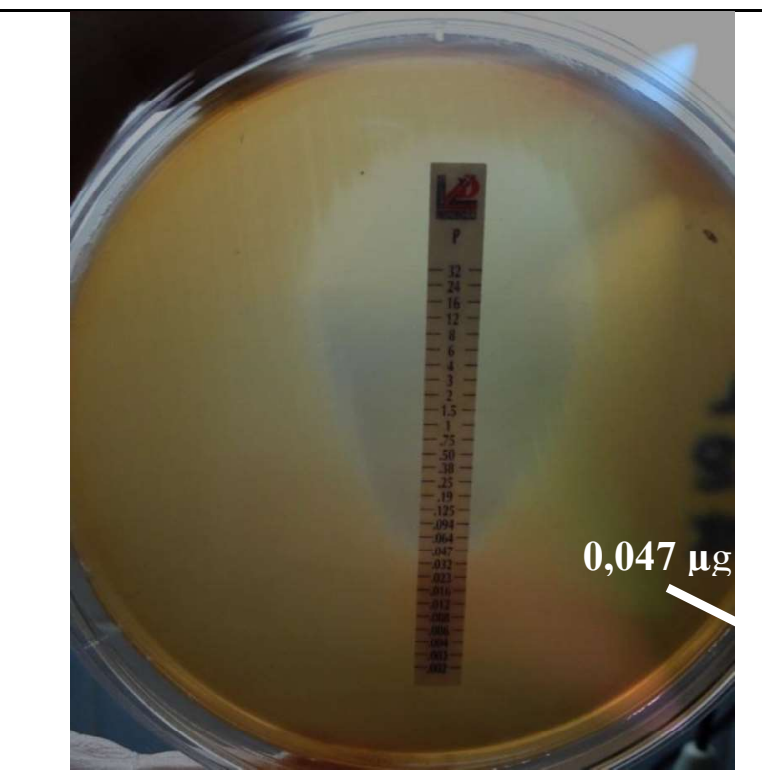

Figura 2. Método Epsilométrico (E-test $\left.{ }^{\circledR}\right)$ para determinación de CIM a penicilina en Streptococcus agalactiae

\section{Discusión}

La determinación in vitro de la CIM permite la detección de resistencia o sensibilidad brindando resultados cuantitativos que se expresan en $\mu \mathrm{g}$ $\mathrm{mL}^{-1}$.

En el presente trabajo encontramos que los resultados de la CIM para el $100 \%$ (96) de las cepas de SGB estudiadas mostraron sensibilidad a la penicilina en concordancia con la bibliografía mundial $(24,25,26)$. Así, un estudio realizado en la República Argentina por Di Bartolomeo y col. ${ }^{(24)}$ sobre 87 cepas de SGB aisladas de embarazadas reportó sensibilidad a penicilina en todos los casos, resultados similares fueron informados por Perez y col. $(2004)^{(25)}$. En Brasil, Dutra y col. ${ }^{(26)}$, estudiaron 430 cepas de diferentes regiones entre 2005 y 2009, de los cuales 249 correspondieron a aislamientos vagino-rectales, también en esa investigación, todos los aislamientos fueron sensibles.

Estos hallazgos difieren de otros investigadores que encontraron sensibilidad disminuida a la penicilina en un $2 \%$ de los casos $^{(17)}$ y Kimura y col. (2008) que hallaron nueve cepas PRGBS entre 1995 y 1998 y cinco más de 159 aislamientos en $2005^{(13)}$. Por otro lado, Dahesh y col. $(2008)^{(14)}$ detectaron cuatro cepas con elevadas CIM a penicilina (todas del serotipo III) de un total de 5631 cepas ensayadas.

Un estudio más reciente en Japón ${ }^{(27)}$ reportó 45 aislamientos de muestras clínicas con PRGBS de un total de $306(14,7 \%)$ entre enero de 2012 y julio de 2013. Estas cepas presentaron, además resistencia a otras drogas.

Las diferencias encontradas con otros autores pueden deberse al origen de las cepas de SGB 
estudiadas. Mientras que las incluidas en este trabajo son cepas colonizantes obtenidas de hisopos vagino-rectales de mujeres embarazadas con 35-37 semanas de su edad gestacional, no hospitalizadas; las otras investigaciones incluyen, además, aislamientos provenientes de esputo, sangre, infecciones de piel y partes blandas y cepas de origen no especificado, aisladas del ambiente hospitalario donde es más frecuente encontrar cepas que han adquirido mecanismos de resistencia $^{(13,14,17,27-31)}$

Estos resultados, obtenidos para la provincia de Misiones, continúan apoyando el uso de penicilina administrada intra-parto, en la prevención de la severa enfermedad neonatal y en el tratamiento de otras infecciones causadas por SGB, en pacientes que no presenten alergia a antimicrobianos beta-lactámicos.

Por otro lado, los hallazgos de tolerancia o sensibilidad disminuida encontradas por autores como Betriu y col. (1994) $)^{(17)}$ Kimura y col. $(2008)^{(13)}$ y Dahesh y col. $(2008)^{(14)}$ impulsan a continuar con las estrategias de vigilancia epidemiológica que permitan detectar sensibilidad disminuida o resistencia a los antibióticos de uso corriente.

\section{Conclusiones:}

En las 96 cepas de SGB estudiadas no se detectó resistencia a penicilina por el método epsilométrico utilizado para la determinación de la concentración inhibitoria mínima, por lo tanto, este antimicrobiano sigue siendo el de elección en la PIP en pacientes que no presentan alergia a los antibióticos beta-lactámicos.

Las condiciones sanitarias y la emergencia de gérmenes con diferentes grados de resistencia a los antibióticos de uso tradicional, tanto en el ámbito hospitalario como de la comunidad, hacen necesaria la vigilancia epidemiológica de la sensibilidad de SGB a penicilina y a otros antimicrobianos de elección en pacientes alérgicas a beta-lactámicos como los macrólidos, para asegurar tratamientos adecuados y alertar sobre nuevos mecanismos de resistencia 0 cepas con sensibilidad disminuida.

Financiación: Este trabajo pudo realizarse gracias a un subsidio otorgado por la Facultad de Ciencias Exactas, Químicas y Naturales de la Universidad Nacional de Misiones (Disposición $\left.\mathrm{N}^{\circ} 2180 / 15\right)$ y a recursos propios.

\section{Bibliografía:}

1. Vergara $M$, Quiroga $M$, , Pegels E, Laczeski M, Oviedo $P$, Rinaldi M. Las infecciones bacterianas y el laboratorio de bacteriología (actualizada y ampliada). $2^{\circ}$ ed. Posadas: Editorial Universitaria. Universidad Nacional de Misiones; 2015. [Book]
2. Kilian M, Greenwood D, Slack R, Peutherer J, Barer M. Medical Microbiology. 17th ed. Amsterdam: Elsevier; 2007.

3. Abdelghany M, Schenferd L. Group B streptococcal infective endocarditis. J Infect Public Health. 2014; 7:237-239. [PubMed] [Free Article]

4. Barcaite E, Bartusevicius A, Tameliene R, Kliucinskas M, Maleckiene L, Nadisauskiene R. Prevalence of maternal group B stretococcal colonisation in European countries. Acta Obstet Gynelcol Scand. 2014; 87:260-271. [PubMed] [Free Article]

5. Schuchat A. Epidemiology of group $B$ streptococcal disease in de the United States: Shifting paradigms. Clin Microbiol Rev. 1998;11:497-513. [PubMed] [Free Article]

6. Hannoun $A$, Shehab M, Khairallah MT, Sabra A, AbiRached $R$, Bazi $T$, et al. Correlation between group $B$ streptococcal genotypes, their antimicrobial resistance profiles and virulence genes among pregnant women in Lebanon. Int J Microbiol. 2009;2009:796512. doi: 10.1155/2009/796512. [PubMed] [Free Article]

7. Edwars MS, Baker CJ. Streptococcus agalactiae. En: Dolin R, Mandell GL. Bennett JE. Enfermedades infecciosas. Principios y prácticas. Vol. 2. Elsevier; 2006. p. 2423-2434.

8. CDC: Center for desease control and prevention. Performance Standards for Antimicrobial Susceptibility Testing; 24th informational Supplement. Atlanta: 2.010. ('accessed 16 April 2015'). [Article]

9. Ministerio de Salud. LegiSalud Argentina. En: http://test.elegis-ar.msal.gov.ar/leisref/public/search.php; consultado el 20/06/2015.

10. Cunningham F G, Gant N, Levenok, Gilstrap III L, Hauth J, Wenstrom K. Williams. Obstetricia. $21^{a}$ ed. Buenos Aires: Editorial Médica Panamericana; 2004.

11. Steele, Russell W. Microbes. En: http://www.antimicrobe.org/b240.asp\#top; consultado el 19/09/2015

12. Laczeski M, Pegels E, Oviedo P, Quiroga M, Vergara M. Streptococcus agalactiae, primer estudio en Misiones de genes de resistencia asociados a serotipos capsulares. Rev. cienc. tecnol. 2013; 20:68-74. [Article] [Free Article]

13. Kimura K, Suzuki S, Wachino J, Kurokawa H, Yamane $K$. Shibata $N$, et al. First molecular characterization of Group B Streptococci with reduced Penicillin Susceptibility. Antimicrob Agents Chemother. 2008;52(8):2890-2897. [PubMed] [Free Article]

14. Dahesh St, Hensler MEt, Van Sorge $N$, Gertz Jr RE, Schrag $S$, Nizet $V$. Point mutation in the Group $B$ Streptococcal PBP2X Gen conferring decreased susceptibility to beta-lactam antibiotics. Antimicrob Agents Chemother. 2008;52(8):2915-2918. [PubMed] [Free Article]

15. CDC: Center for Disease Control and Prevention Prevention of perinatal group B streptococcal desease. MMWR Morbid Mortal Wkly Rep. 2002;1-22. [Article]

16. Smaill F. Intrapartum antibiotics for group $B$ streptococcal colonization. Cochrane Database of Sistematic Reviews. 1999;3:1-5. [PubMed] [Free Article]

17. Betriu C, Gómez M, Sánchez A, Cruceyra A, Romero J, Picazo J. Antibiotic Resistance and Penicillin Tolerance in Clinical Isolates of Group B Streptococci. Antimicrob Agents Chemother. 1994;38(9):2183-2186. [PubMed] [Free Article]

18. Kimura K, Nagano N, Arakawa Y..Classification of group $B$ Streptococci with reduced B-lactam susceptibility (GBS-RBS) based on the amino acid substitution in PBPs. J Antimicrob Chemother. 2015;70(6):1601-1603. [PubMed] [Free Article]

19. Florez J. Farmacología Humana. $3^{\circ} \mathrm{ed}$. Barcelona:Masson S.A.;1997. 
Sensibilidad a penicilina en SGB.

20. Procop G, Woods G, et al. Resistencia bacteriana a los antibióticos. En: Winn W, Allen S, Janda W, Doneman E. Diagnóstico microbiológico. $6^{a}$ ed. Buenos Aires: Médica Panamericana; 2008. p. 902-919.

21. MacFaddin JF. Pruebas Bioquímicas para la identificación de bacterias de importancia clínica. Buenos Aires: Editorial Médica Panamericana; 2003.

22. CLSI: Clinical and Laboratory Standards Institute. Methods for Antimicrobial Dilution and Disk Susceptibility Testing of Infrequently Isolated or Fastidious Bacteria. Wayne PA: CLSI Guideline M45, 2015.

23. CLSI: Clinical and Laboratory Standards Institute. Reference Methods vs Commercial Methods and CLSI vs US Food and Drug Administratrion Interpretive Criteria (Breakpoints). Tabla 2H-1 Streptococcus spp. BHemolytic Group $\mathrm{M02}$ and M07. M100S. 26th ed. Atlanta; 2016.

24. Di Bartolomeo S, Gentile M, Priore G, Valle S, Di Bella A. Streptococcus agalactiae in pregnant women. Prevalence at the Posadas Hospital. Rev. Argent. Microbiol. 2005;37(3):142-144. [PubMed]

25. Perez J, Limansky A, Toresani I, Ebner G, Di Bartolomeo $S$, De Inocenti l, et al. Distribución de tipo capsular y sensibilidad antimicrobiana de Streptococcus agalactiae productores de infecciones en Argentina. Rev. Argent. Microbiol. 2004;36:63-67. [PubMed]

26. Dutra V, Alves V, Olendzki A, Dias C, Bastos de A, Santos $G$, et al. Streptococcus

agalactiae in Brazil: serotype distribution, virulence determinants and antimicrobial susceptibility. BMC Infect Dis, 2014;14:323. doi:10.1186/1471-2334-14-323. [PubMed] [Free Article]

27. Seki T, Kimura K, Reid ME, Miyazaki A, Banno H, Jin W, et al. High isolation rate of MDR group streptococci with reduced penicillin susceptibility in Japan. J Antimicrob Chemother. 2015;70:2725-2728. [PubMed] [Free Article]

28. Barros $R$, Jobst R, Souza A F de, Melo A L de, Modino S $S B$ de. Evaluation of Streptococcus agalactiae carriage among high risk pregnant women attended in Niterói, RJ, Brazil. Rev. Patol. Trop. 2015;44(4):386-394.

29. Crespo-Ortiz M, Catañeda-Ramirez C, Recalde-Bolaños $M$, Vélez-Londoño J. Emerging trends in invasive and noninvasive isolates of Streptococcus agalactiae in a Latin American hospital: a 17-year study. BMC Infectious Diseases. 2014;14:1-11. [Article]

30. Kasahara K, Baltus AJ, Lee S-H, Edelstein MA, Edelstein PH. Prevalence of Non-Penicillin-Susceptible Group B Streptococcus in Philadelphia and Specificity of Penicillin Resistance Screening Methods. J Clin Microbiol. 2010;48(4):1468-1469. [PubMed] [Free Article]

31. Nagano N, Nagano Y, Toyama M, Kimura K, Tamura T, Shibayama $K$, et al. Nosocomial spread of multidrogresistant group $B$ streptococci with reduced penicillim susceptibility belonging to clonal complex 1. Antimicrob Chemother. 2012;67:849-856. [PubMed] [Free Article] 